\title{
AVALIAÇÃO DE UM TANQUE DE DECANTAÇÃO DE SÓLIDOS UTILIZANDO TLUIDODINÂMICA COMPUTACIONAL
}

\author{
E. F. S. PEREIRA e L. M. N de Gois
}

Universidade Federal da Bahia, Escola Politécnica, Departamento de Engenharia Química

E-mail para contato: elcio.pereirasicm@gmail.com

RESUMO - O presente trabalho aborda o problema da separação de sólidos de líquido, em um tanque de decantação que trata um efluente industrial. Foram coletadas amostras ao longo do comprimento do tanque para a caracterização dos sólidos e construído um modelo para simulação fluidodinâmica, do qual foram extraídas amostras equivalentes às reais para validação do modelo utilizando o Teste Estatístico de Hipótese como critério de validação.

\section{INTRODUÇÃO}

O tanque de decantação em estudo consta de um clarificador industrial que foi dimensionado para cumprir as exigências ambientais com relação ao teor de sólidos em suspensão no fluido clarificado. Como o balanço de massa é o objeto de interesse neste tipo de equipamento, o comportamento dos sólidos no tanque é normalmente ignorado. Daí, a avaliação dos parâmetros de operação apresenta como principal dificuldade a mínima margem disponível para variações, além do elevado custo para realizar ações desta natureza. Com isso, a modelagem numérica apresenta como principal vantagem a flexibilidade de manipulação de parâmetros operacionais, além da grande quantidade de informações disponíveis como resposta aos cálculos. Neste contexto o objetivo do presente trabalho é o de realizar uma avaliação do tanque de decantação industrial em operação normal, obtendo respostas que irão auxiliar na melhor operação e manutenção do tanque. Será utilizada a estratégia de combinar a abordagem numérica com a abordagem experimental de maneira complementar, utilizando um método estatístico para validação do modelo.

\section{O TRABALHO EXPERIMENTAL}

A parte experimental do presente trabalho consta, basicamente, da tomada de amostras no em um tanque de sedimentação, em escala industrial, com dimensões de 38 metros de largura, 98 metros de comprimento e $2,95 \mathrm{~m}$ de profundidade que está localizado na unidade de tratamento de efluentes da empresa Dow Química/BA. Esta unidade é alimentada através de uma corrente com vazão de $1979,16 \mathrm{~m}^{3} / \mathrm{h}$, com composição predominantemente formada por água, $\mathrm{CaCO}_{3}$, sílica e alguns sais solúveis. Na Figura 1 são mostradas fotos da unidade de tratamento e do duto de alimentação. 


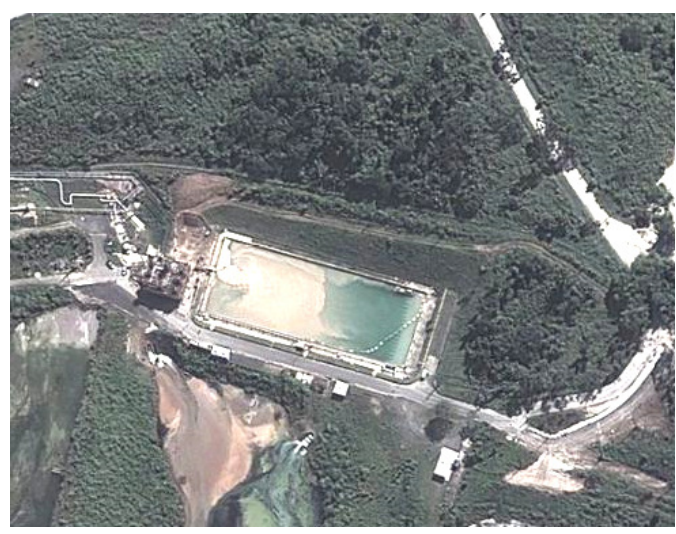

Figura 1 - Vista aérea da unidade

As amostras foram todas coletadas com o auxílio de uma 'draga" que, geralmente, é utilizada na limpeza do fundo do tanque, onde foram coletadas 10 amostras em duplicata, em um comprimento de $66 \mathrm{~m}$, que foi dividido em espaçamentos médios de 6,6m, na área escolhida, pois, foram excluídas as regiões de entrada e de saída do tanque. Na figura 2-a) é mostrada a área de medição do tanque e na figura 2-b) uma ilustração da operação da "draga".

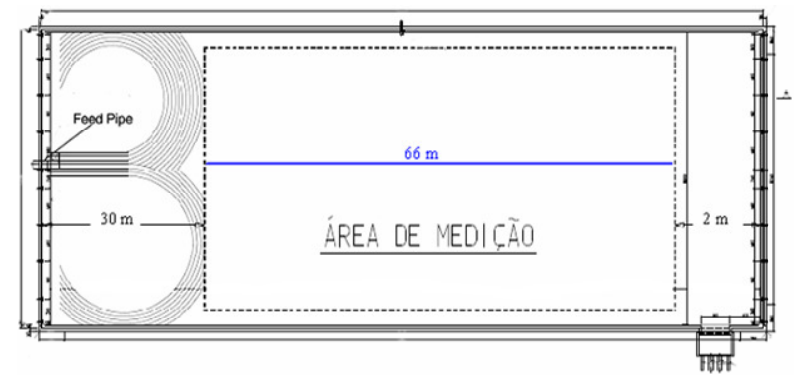

Figura 2 - Área de medição e amostragem,

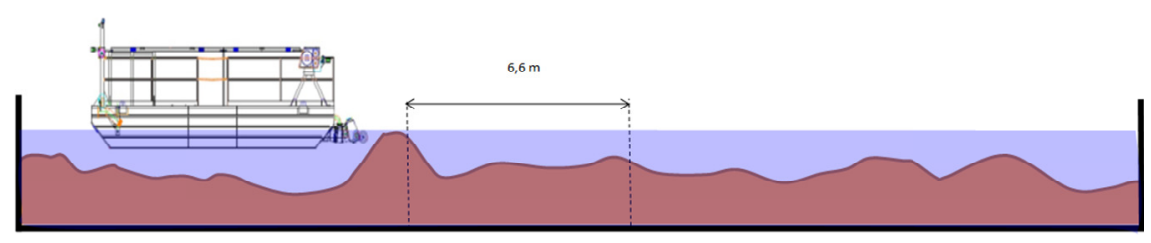

Figura 3 - Ilustração da operação da draga

Como para a avaliação do tanque era importante também conhecer o perfil do acúmulo de sólidos ao longo do seu comprimento, foi utilizada uma "boia de interface" com peso suficiente para afundar na água, porém não o suficiente para superar o empuxo da lama. A bóia pesava $0,75 \mathrm{~kg}$ e possuía uma geometria adequada que permitia que ela fosse sempre posicionada na 
interface sólido-líquido. A figura 3 mostra o procedimento de medição da altura da lama existente no tanque. Deve-se salientar que altura máxima medida com a bóia de interface foi de 3,03 $\mathrm{m}$ e que nos 30 primeiros metros de comprimento do tanque em relação ao ponto de alimentação, a altura da lama não foi medida, porque a agitação da corrente alimentada não permitia o repouso da bóia sobre a camada de sólidos. Os dados obtidos com os experimentos estão na tabela 1 .

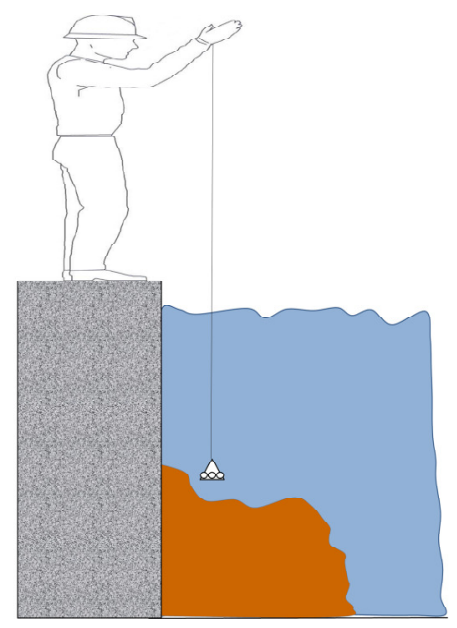

Figura 4 - Detalhes de como utilizar a bóia de interface

Tabela 1 - Dados obtidos das amostras e das medições no tanque.

\begin{tabular}{|c|c|c|c|c|}
\hline Amostra no $^{\mathbf{0}}$ & $\begin{array}{c}\text { Distância do ponto } \\
\text { de alimentação }(\mathbf{m})\end{array}$ & $\begin{array}{c}\text { Altura da interface } \\
\text { sólido-líquido }(\mathbf{m})\end{array}$ & $\begin{array}{c}\text { Faixa de coleta de } \\
\text { amostras }(\mathbf{m})\end{array}$ & $\begin{array}{c}\text { Concentração mássica } \\
\text { dos sólidos }(\boldsymbol{\%})\end{array}$ \\
\hline 1 & 30 & 2,85 & $30-37$ & 8,13 \\
\hline 2 & 37 & 2,13 & $37-48$ & 9,16 \\
\hline 3 & 48 & 2,28 & $48-54$ & 9,35 \\
\hline 4 & 54 & 2,28 & $54-60$ & 9,74 \\
\hline 5 & 60 & 2,15 & $60-66$ & 10,3 \\
\hline 6 & 66 & 2,05 & $66-72$ & 7,17 \\
\hline 7 & 72 & 1,96 & $72-78$ & 6,74 \\
\hline 8 & 78 & 1,77 & $78-84$ & 6,71 \\
\hline 9 & 84 & 1,53 & $84-90$ & 5,79 \\
\hline 10 & 90 & 1,35 & $90-96$ & 4,73 \\
\hline- & 96 & 1,17 & - & \\
\hline
\end{tabular}

\section{A SIMULAÇÃ̃O}

A simulação do presente trabalho foi realizada através da fluidodinâmica computacional empregando-se a abordagem Euler-Lagrange aplicada ao modelo de fase discreta, onde a fase sólida deve ser tratada como dispersa e a trajetória das partículas obtida após a resolução da 
equação de movimento para cada partícula. Barreira, (2003). Este modelo foi o escolhido por dar como resposta as distribuições granulométricas da fase sólida e as trajetórias das partículas no escoamento, ressaltando a influência dos contornos rígidos. Segundo, Dickenson e Ssansalone (2009) as equações da modelagem para o modelo de fase discreta são as seguintes:

$$
\begin{gathered}
\vec{v}_{n-1}-\vec{v}_{n}+\left(\frac{d \vec{v}}{d t}\right) \cdot \Delta t \\
\frac{d v_{s}}{d t}=F_{D} \cdot\left(v_{l}-v_{s}\right)+\frac{g_{x} \cdot\left(\rho_{s}-\rho_{l}\right)}{\rho_{s}}
\end{gathered}
$$

Onde $\mathrm{F}_{\mathrm{D}}$ é a força de arraste, $g_{x}$ é a aceleração da gravidade $\left(\mathrm{m} / \mathrm{s}^{2}\right) \rho$ é a massa específica. os índices (l) e (s) são utilizados para distinguir a fase líquida da fase sólida, $\vec{v}_{n-1}$ é a velocidade da partícula na posição n-1 (m/s) e $\Delta$ t é o passo de integração (s). A aceleração das partículas são obtidas através do balanço de forças, Dickenson e Sansalone (2009); Barreira, (2003). Na simulação foram utilizadas como condições de fronteira os dados contidos na Tabela 1 mostrada a seguir e, para a validação, os dados simulados foram comparados com os resultados experimentais encontrados. Ou seja, foram definidos 10 planos e, cada um deles, comparados com as amostras obtidas experimentalmente.

Tabela 2 - Dados de entrada para a simulação

\begin{tabular}{|c|c|c|c|c|c|}
\hline Materiais & $\begin{array}{c}\text { Temperatura } \\
\left({ }^{\circ} \mathbf{C}\right)\end{array}$ & $\begin{array}{c}\text { Massa Específica } \\
\left(\mathbf{k g} / \mathbf{m}^{\mathbf{3}}\right)\end{array}$ & $\begin{array}{c}\text { Viscosidade } \\
(\mathbf{c P})\end{array}$ & $\begin{array}{c}\text { Concentração } \\
(\mathbf{p p m})\end{array}$ & Vazão (kg/s) \\
\hline$\left(\mathrm{H}_{2} \mathrm{O}\right)$ & 60 & 1027 & 1,0003 & N/A & 373,25 \\
\hline$\left(\mathrm{CaCO}_{3}\right)$ & 60 & 2707,41 & - & 950 & 0,3454 \\
\hline
\end{tabular}

\section{RESULTADOS E DISCUSSÃO}

Os resultados obtidos referentes às amostras experimentais retiradas do tanque e as simuladas foram comparadas através de gráficos, onde são representadas as suas distribuições granulométricas. As Figuras 4 e 5 são histogramas de distribuição de massa em relação à faixas de diâmetro das partículas. Com estes é feita uma comparação referente à primeira amostra real coletada e a primeira amostra da simulação, onde se percebe claramente maior acúmulo de massa na faixa de diâmetros menores para esta amostra. 


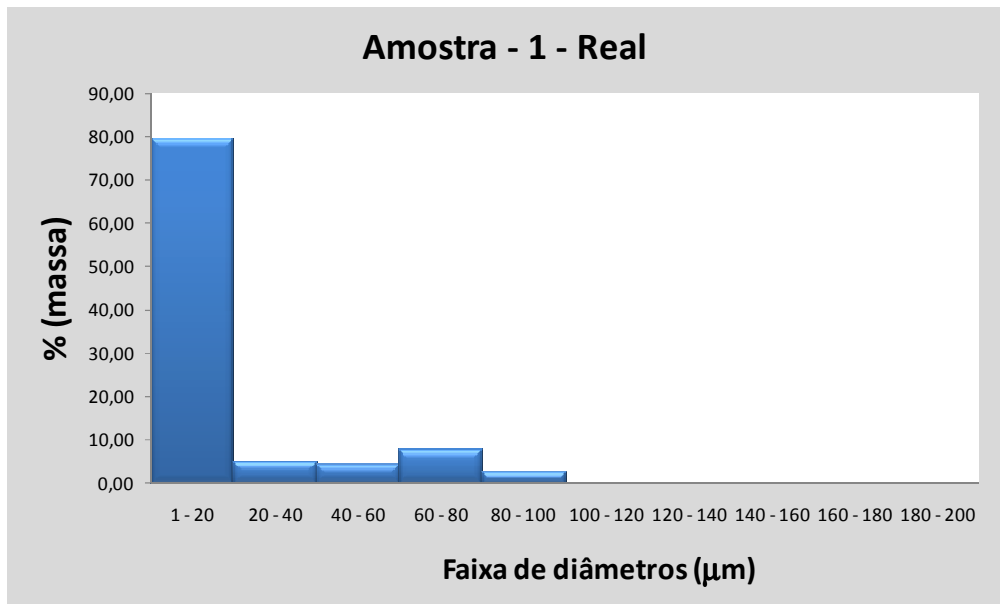

Figura 4 - Distribuição granulométrica da $1^{\mathrm{a}}$ amostra real

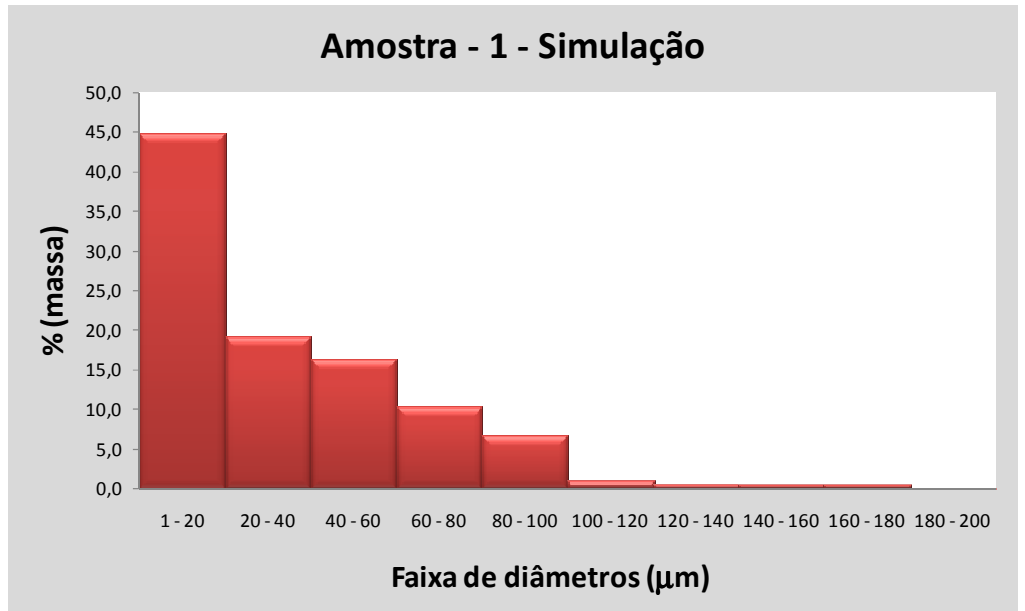

Figura 5 - Distribuição granulométrica da $1^{\mathrm{a}}$ amostra da simulação

\section{VALIDADAÇÃO DO MODELO ADOTADO NA SIMULAÇÃO - TESTE DE HIPÓTESE: DUAS AMOSTRAS t.}

Com o objetivo de validar os valores obtidos na simulação, utilizou-se o teste de hipótese de duas amostras t, que foi uma generalização desenvolvida por Welch (1947) utilizando a distribuição t criada por Gosset (1908). O teste tem como objetivo básico o confronto de valores amostrais efetivos com parâmetros populacionais hipotéticos. Segundo Welch (1947), este teste busca provar que se a média populacional de duas amostras independentes são iguais, as mesmas pertencem à mesma população, Para validar os dados da simulação a esta condição tem que ser 
atendida, ou seja, a média populacional da amostra real $\left(\mu_{\text {real }}\right)$ é igual à média populacional da amostra retirada da simulação $\left(\mu_{\text {teórico }}\right)$. Ou seja:

$$
\mu_{\text {real. }}=\mu_{\text {teốriro }}
$$

E a hipótese será aceita se o valor da distribuição de probabilidade amostral calculada, $t_{\text {calculado }}$ esteja dentro do intervalo de aceitação definido por:

$$
\left(-t_{\text {crítico }}<t_{\text {calculado }}<+t_{\text {crítico }}\right)
$$

Conforme mostrado na Figura 5, onde, $-\mathrm{t}_{\text {crítico }} \mathrm{e}+\mathrm{t}_{\text {crítico }}$ são os valores dos extremos da curva de distribuição de probabilidade amostral $t$, e indicam os limites onde o teste de hipótese pode ser considerado verdadeiro. Os valores críticos de t dependem do nível de significância do teste $(\alpha)$ que é um valor de probabilidade fixo, abaixo do qual a hipótese é considerada falsa. $\mathrm{O}$ valor de $5 \%$ é um valor típico de $\alpha$, mas com a finalidade de ser mais conservativo, foi considerado $\alpha=25 \%$.

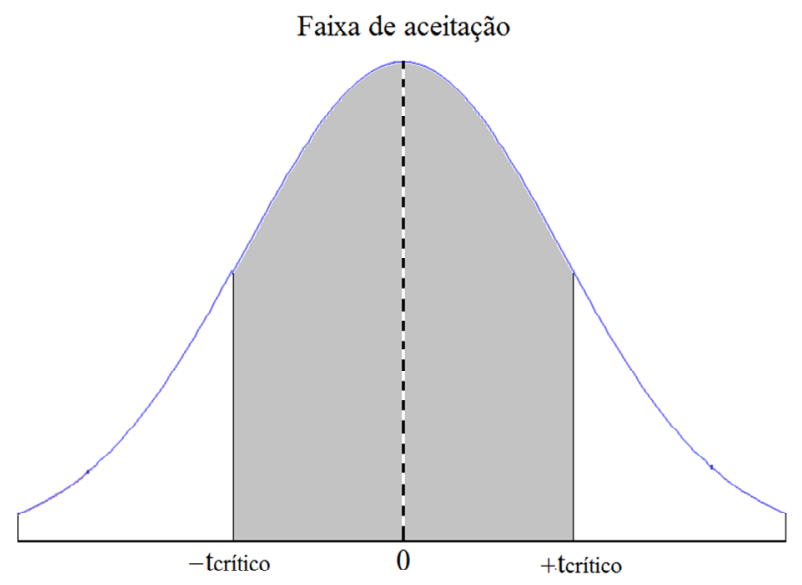

Figura 5 - Curva de distribuição amostra t, com seu intervalo de aceitação do teste.

Segundo Welch (1947), o cálculo do t calculado pode ser feito através da equação (6).

$$
t_{\text {calculado }}=\frac{\left(\bar{X}_{\text {real }}-\bar{X}_{\text {teórico }}\right)-\left(\mu_{\text {real }}-\mu_{\text {teórico }}\right)}{\hat{\sigma}_{\bar{X}_{\text {rsal }}-\bar{X}_{\text {teórico }}}}
$$

Onde: 
- $\bar{X}_{\text {real }}$ e $\bar{X}_{\text {teórico }}$, são as médias dos diâmetros das amostras de lama real e as obtidas da simulação, respectivamente.

- $\hat{\sigma}_{\bar{X}_{1}-\bar{X}_{2}}$ o erro padrão que pode ser calculado através da equação (7).

$$
\hat{\sigma}_{R_{\text {rsal }}-\Omega_{\text {tabrico }}}=S_{P} \cdot \sqrt{\frac{1}{n_{\text {real }}}+\frac{1}{n_{\text {teórico }}}}
$$

Onde $n_{\text {real }}$ e $n_{\text {teórico }}$ representam o tamanho das amostras de lama, real e a obtida com a simulação e o $S_{P}$ é calculado utilizando a equação (8).

$$
S_{P}=\sqrt{\frac{\left(n_{\text {real }}-1\right) \cdot S_{\text {real }}^{2}+\left(n_{\text {térico }}-1\right) \cdot S_{\text {teórico }}^{2}}{n_{\text {real }}-n_{\text {teórico }}-2}}
$$

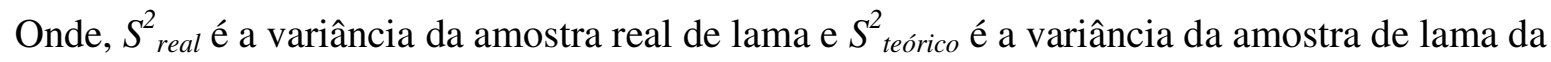
simulação. Na Tabela 3 são mostrados e comparados os valores de $t_{\text {crítico }}$ e de $t_{\text {calculado }}$ obtidos para todas as amostras experimentais coletadas.

Tabela 3 - Resultados

\begin{tabular}{|c|c|c|c|c|}
\hline Amostra & $\mathbf{t}_{\text {crítico }}$ & $\mathbf{t}_{\text {calculado }}$ & $\begin{array}{c}\text { Valor da probabilidade } \\
\mathbf{P} \text { (unicaudal) }\end{array}$ & $\begin{array}{c}\text { Concentração mássica dos sólidos } \\
(\%)\end{array}$ \\
\hline 1 & $\pm 0,684$ & $2,27 \mathrm{E}-16$ & 0,5 & 8,13 \\
\hline 2 & $\pm 0,684$ & $-3,96 \mathrm{E}-16$ & 0,5 & 9,16 \\
\hline 3 & $\pm 0,683$ & $-3,45 \mathrm{E}-16$ & 0,5 & 9,35 \\
\hline 4 & $\pm 0,683$ & 0 & 0,5 & 9,74 \\
\hline 5 & $\pm 0,684$ & 0 & 0,5 & 10,3 \\
\hline 6 & $\pm 0,685$ & 0 & 0,5 & 7,17 \\
\hline 7 & $\pm 0,684$ & $1,79 \mathrm{E}-16$ & 0,5 & 6,74 \\
\hline 8 & $\pm 0,683$ & $-3,21 \mathrm{E}-16$ & 0,5 & 6,71 \\
\hline 9 & $\pm 0,683$ & $3,29 \mathrm{E}-16$ & 0,5 & 5,79 \\
\hline 10 & $\pm 0,683$ & $-3,21 \mathrm{E}-16$ & 0,5 & 4,73 \\
\hline
\end{tabular}

Observando-se os valores do $t_{\text {calculado }}$ mostrados na tabela 3 , verifica-se que todos os valores obtidos se situaram na vizinhança de zero, ou seja, no centro da faixa de aceitação do problema (expressão 5). O termo da probabilidade $\mathrm{P}$, representa a área delimitada pelo valor de $\mathrm{t}_{\text {calculado, }}$ ou seja, como os valores de $t_{\text {calculado, }}$, foram praticamente iguais a zero, o valor de $\mathrm{P}$ fica sendo igual à área da metade da curva Gaussiana. A comparação entre o valor de $\mathrm{P}$ e o nível de significância do teste também como critério de aceitação. Com base nos dados apresentados na tabela 3 conclui-se que 
todos os dez testes de hipótese foram aceitos e que a hipótese e que a simulação reflete de maneira fiel o escoamento no tanque real.

\section{CONCLUSÕES.}

A Fluidodinâmica Computacional empregando-se a abordagem Euler-Lagrange aplicada ao modelo de fase discreta, consegue reproduzir de forma convincente o comportamento do tanque de decantação industrial estudado. Quanto à aplicação do teste de duas amostras t como critério de validação, conclui-se que ele poder ser perfeitamente utilizado em escala industrial, porque o mesmo apresenta fácil aplicação e não está limitado ás condições de entrada e saída, como por exemplo a eficiência de separação ou balanço de massa. Por último, conclui-se também que este tipo de avaliação pode trazer reduções significativas de custos operacionais e de manutenção em tanques de decantação industriais e que após a validação da simulação, é aberto o precedente para outras simulações em condições de operação diversas, o que possibilita utilizar os resultados para implementa melhorias na forma de operar e na manutenção do tanque, por exemplo, a elaboração de um plano de dragagem do tanque inteligente e bem mais preciso do que ocorre atualmente.

\section{REFERÊNCIAS}

BARREIRA, M. N. Introdução à modelagem em 3D para reatores "Air-Lift" empregando o método dos volumes finitos na resolução da fluidodinâmica (CFD). Universidade Estadual de Campinas, p. 263, 20032003.

DICKENSON, J. A.; SANSALONE, J. J. Discrete Phase Model Representation of Particulate Matter (PM) for Simulating PM Separation by Hydrodynamic Unit Operations. Environmental Science \& Technology: American Chemical Society. 43: 8220-8226 p. 2009.

GOSSET, W. S. The probable error of a mean. Biometrika, v. 6, n. 1/25, 19081908.

WELCH, B. L. The Generalization of "Student's" problem when several different population variances are involved. Biometrika, v. 34, n. 1/2, p. 28-35, 19471947. 